\section{Abrechnungsfähigkeit strahlentherapeutischer Leistungen durch Facharzt für Diagnostische Radiologie}

Die Judikatur musste sich wiederholt mit der Frage befassen, ob und in welchem Umfang zugelassene Fachärzte diagnostische Leistungen in der vertragsärztlichen Versorgung erbringen und abrechnen können (vgl. etwa Fortschr Röntgenstr 2006, 132; 2006, 349; 2011, 401; 2011, 486; 2013, 775; 2014, 187; 2014, 889; abrufbar unter http://radiologie-und-recht.de/de/archiv. radiologie.und.recht/beitraege.ab.2003/). In einer aktuellen Entscheidung hatte das BSG (Urt. v. 4.5.2016, Az.: B 6 KA 13/15R) nunmehr darüber zu befinden, ob ein Facharzt für Diagnostische Radiologie strahlentherapeutische Leistungen zu Lasten der Gesetzlichen Krankenversicherung erbringen und abrechnen darf.

\section{Sachverhalt}

Der Kläger, ein seit dem 30.12.1993 zur vertragsärztlichen Versorgung zugelassener Facharzt für Diagnostische Radiologie, begehrte die Genehmigung zur Abrechnung der strahlentherapeutischen Leistungen nach den Gebührenordnungspositionen (GOP) 25310 (Weichstrahl- oder Orthovolttherapie) und 25340 (Bestrahlungsplanung für die perkutane Bestrahlung ohne Rechnerunterstützung und individuelle Dosisplanung) des EBM-Ä. Die beklagte Kassenärztliche Vereinigung (KV) erteilte zwar die Genehmigung zur Durchführung strahlentherapeutischer Leistungen, versagte aber zugleich deren Abrechnungsfähigkeit mit der Begründung, dass strahlentherapeutische Leistungen für den Kläger wegen fehlender Fachgebietszugehörigkeit fachfremd seien. Die Tätigkeit eines Radiologen mit Zulassung für Diagnostische Radiologie oder für Radiologische Diagnostik müsse auf die diagnostischen Leistungen beschränkt sein. Sowohl im erst-, als auch im zweitinstanzlichen Verfahren ist der Kläger erfolglos geblieben.

\section{Versagung der Abrech- nungsberechtigung}

In der Revisionsinstanz kam das BSG ebenfalls zu dem Schluss, dass ein Facharzt für Diagnostische Radiologie keinen Anspruch auf Abrechnung strahlentherapeutischer Leistungen hat.

\section{Erfüllung der fachlichen Vorausset- zungen nach der Vereinbarung zur Strahlendiagnostik und -therapie}

Der erkennende Senat stellte zunächst fest, dass der klagende Facharzt für Diagnostische Radiologie die besonderen Voraussetzungen, unter denen die Qualitätssicherungsvereinbarungen zur Strahlendiagnostik und -therapie ungeachtet einer einschlägigen Facharztweiterbildung eine Leistungserbringung ermöglicht, nicht erfüllt.

§135 Abs. 2 S. 1 SGB V ermächtigt die die Kassenärztliche Bundesvereinigung gemeinsam mit dem Spitzenverband Bund der Krankenkassen, in sog. Vereinbarungen Qualitätsvoraussetzungen für ärztliche und Leistungen, die wegen der Anforderungen an ihre Ausführung oder wegen der Neuheit des Verfahrens

- besonderer Kenntnisse und Erfahrungen (Fachkundennachweis),

- einer besonderen Praxisausstattung oder

- weiterer Anforderungen an die Versorgungsqualität bedürfen,

einheitliche Voraussetzungen für die Ausführung und Abrechnung dieser Leistungen zu regeln (vgl. Fortschr Röntgenstr 2012, 848). Von dieser Ermächtigung haben die Vertragspartner für den Bereich der Strahlentherapie in Form der Vereinbarung zur Strahlendiagnostik und -therapie (StrahlendiagV) Gebrauch gemacht. Nach § 2 StrahlendiagV ist die Ausführung und Abrechnung von Leistungen der Strahlentherapie im Rahmen der vertragsärztlichen Versorgung erst nach Erteilung der Genehmigung durch die Kassenärztliche Vereinigung zulässig. Der antragstellende Arzt hat einen Anspruch auf deren Erteilung, wenn er die Voraussetzungen der fachlichen Befähi-

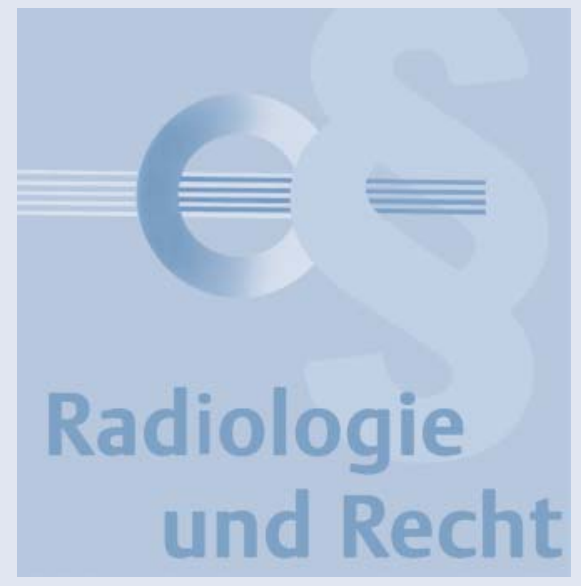

gung ( $\$ 9$ StrahlendiagV) sowie der apparativen Ausstattung ( $§ 12$ StrahlendiagV einschließlich Anlage II) erfüllt.

Nach Ansicht des BSG sind die jeweiligen Tatbestände des $§ 9$ StrahlendiagV im vorliegenden Fall allesamt nicht erfüllt. In Bezug auf die erforderlichen Kenntnisse und Fähigkeiten ist nicht auf die derzeit gültige Fassung der Weiterbildungsbildungsordnung, sondern auf die geltende Fassung zum Zeitpunkt der tatsächlich erworbenen Qualifikationen abzustellen, da eine spätere formale Änderung des Gesetzes eine fehlende Qualifikation nicht ersetzen kann. Nach § 9 Abs. 1 lit. a StrahlendiagV gilt die fachliche Befähigung für die Ausführung und Abrechnung von Leistungen der Nahbestrahlungs-, Weichstrahl- und Orthovolttherapie als gegeben, sofern der betreffende Arzt zum Führen der Facharztbezeichnung

- „Facharzt für Strahlentherapie“,

- „Facharzt für Radiologie, Teilgebiet: Strahlentherapie“ (nach Übergangsrecht der WBO) oder

- „Facharzt für Radiologie“ (sofern er die fachliche Qualifikation für die Strahlentherapie erworben hat)

berechtigt ist. Da der Kläger kein Facharzt für Radiologie, sondern Facharzt für Radiologische Diagnostik/Diagnostische Radiologie ist, greift dieser Ausnahmetatbestand nicht.

Der betreffende Arzt erfüllt auch die Voraussetzungen nach $\S 9$ Abs. 1 lit. b StrahlendiagV nicht. Danach liegt die erforderliche fachliche Qualifikation gleichsam vor, wenn eine unter $\S 9$ Abs. 1 lit. a StrahlendiagV genannte Facharztbezeichnung zwar nicht erworben wurde, der Nachweis 
einer entsprechenden Weiterbildung in fachgebietsspezifischer Nahbestrahlungs-, Weichstrahl- und Orthovolttherapie aber durch die Vorlage ausreichender Zeugnisse erbracht wurde und die Weiterbildungsordnung für diese Weiterbildung den Erwerb eingehender Kenntnisse, Erfahrungen und Fertigkeiten vorschreibt.

Nach § 9 Abs. 1 lit. c StrahlendiagV sind die fachlichen Voraussetzungen ferner erfüllt, wenn eine Weiterbildung nach Absatz 1a oder 1b nicht stattgefunden hat, aber Zeugnisse

- für die Nahbestrahlungstherapie eine mindestens sechsmonatige ständige Tätigkeit in dieser Strahlentherapie unter der Leitung eines dazu ermächtigten Arztes,

- für die Weichstrahltherapie eine mindestens sechsmonatige ständige Tätigkeit in der Strahlentherapie von Hautkrankheiten unter der Leitung eines dazu ermächtigten Arztes und

- für die Orthovolttherapie eine mindestens zwölfmonatige ständige Tätigkeit in dieser Strahlentherapie unter der Leitung eines dazu ermächtigten Arztes vorgelegt werden. Dass der Kläger diese Vorgaben erfüllt hat, war nach Auffassung des Gerichts in dem zugrunde liegenden Fall nicht ersichtlich. Zudem fehlte es an einem Nachweis für die Ableistung eines Kolloquiums nach §9 Abs. 4 StrahlendiagV.

\section{Beachtung der Fachgebietsgrenzen}

Das BSG gelangte - in Übereinstimmung mit den Vorinstanzen - außerdem zu dem Ergebnis, dass die Genehmigung der Durchführung und Abrechnung strahlentherapeutischer Leistungen (jedenfalls) wegen ihrer Fachfremdheit bezogen auf einen Facharzt für Diagnostische Radiologie grundsätzlich ausscheidet.

Die Heilberufs- bzw. Kammergesetze der Länder sowie die auf Grundlage dieser Gesetze von der Landesärztekammer erlassenen Weiterbildungsordnungen normieren die Verpflichtung derjenigen Ärzte, die eine Gebietsbezeichnung führen, ihre Tätigkeit auf dieses Fachgebiet zu beschränken (z. B. §41 Abs. 1 HeilberufsG NW). Das BSG geht in ständiger Rechtsprechung davon aus, dass die Einhaltung der Fachgebietsgrenzen auch für die Tätigkeit des Arztes in der vertragsärztlichen Versor- gung gilt. Die Regelungen des Vertragsarztrechts zur Zulassung ( $\S 18$ Abs. 1 S. 2 und $\S 24$ Abs. 6 Ärzte-ZV), zur Bedarfsplanung (§ 101 Abs. 1 S. 5 SGB V und § 12 Abs. 7 Ärzte-ZV) und zu den Zulassungsbeschränkungen (§103 Abs. 2 S. 3 SGB V) sowie zur Gliederung der in der fachärztlichen Versorgung abrechenbaren Leistungen nach den einzelnen Facharztgruppen ( $\$ 87$ Abs. 2a S. 1 SGB V) belegen in ihrer Zusammenschau, dass der Gesetzgeber von einer nach einzelnen ärztlichen Fachgebieten gegliederten ambulanten vertragsärztlichen Versorgung ausgeht (vgl. BSG, Urt.v. 22.3.2006, Az.: B 6 KA 75/04 R). Ein gegliedertes Facharztwesen mit einer arztgruppenbezogenen Bedarfsplanung und entsprechenden Zulassungsbeschränkungen, die ebenfalls auf die jeweilige Arztgruppe zugeschnitten sind, kann seine Funktion nicht erfüllen, wenn jeder Facharzt auf jedem Gebiet Leistungen ohne Einschränkungen erbringen und abrechnen kann. Das BSG betonte in diesem Kontext, dass damit jede nicht nur ausnahmsweise, sondern systematische Leistungserbringung außerhalb des Fachgebiets ausgeschlossen ist.

Wegen ihres Eingriffs in das Grundrecht der Berufsfreiheit nach Art. 12 Abs. 1 GG sind der Beschränkung auf die Fachgebietsgrenzen rechtliche Grenzen gesetzt. Sie ist daher nur rechtmäßig, soweit die betroffenen Leistungen für das Fachgebiet nicht wesentlich und nicht prägend sind, die Abgrenzung vom fachlich medizinischen Standpunkt aus sachgerecht ist und der Facharzt in der auf sein Fachgebiet beschränkten Tätigkeit eine ausreichende Lebensgrundlage finden kann (BSG MedR 2005, 364). Nach § 2 Abs. 2 S. 2 WBO bestimmt die Gebietsdefinition die Grenzen für die Ausübung der fachärztlichen Tätigkeit, welche nicht durch Zusatzweiterbildungen erweitert werden können (§2 Abs. 4 S. 4 WBO). Für die Beurteilung, ob Leistungen fachzugehörig oder fachfremd sind, ist darauf abzustellen, welche Inhalte und Ziele der Weiterbildung für das jeweilige Fachgebiet in der Weiterbildungsordnung genannt werden und in welchen Bereichen eingehende Kenntnisse, Erfahrungen und Fertigkeiten erworben werden müssen. Schwerpunkt- oder Zusatzbezeichnungen haben keinen Einfluss auf die Fachfremdheit einer Leistung. Ebenso sind individuelle Qualifikationen für die Zuord- nung bestimmter Leistungen zu einem Fachgebiet irrelevant; die Fachzugehörigkeit bemisst sich allein nach den allgemein der Fachgruppe zugeordneten Weiterbildungsinhalten, die in der jeweiligen Weiterbildungsordnung des Landes festgelegt werden. Abzustellen ist wiederum auf die zur Zeit des Erwerbes der Approbation des betreffenden Arztes geltende Fassung der Weiterbildungsordnung des jeweiligen Kammerbezirkes.

Zur Beurteilung des vorliegenden Falles war die Weiterbildungsordnung der Ärztekammer Nordrhein maßgeblich. Während diese vor 1988 für Radiologen noch „eingehende Kenntnisse und Erfahrungen in der Strahlentherapie und im Strahlenschutz" verlangte, enthielt die Weiterbildungsordnung vom 1.7.1988 als Inhalt und Ziel der Weiterbildung zum Arzt für Radiologische Diagnostik nur noch „eingehende Kenntnisse und Erfahrungen im Strahlenschutz sowie Kenntnisse in der Indikation zur Strahlentherapie“. Das frühere Teilgebiet „Strahlentherapie" bildete nun eine eigene Facharztgruppe. Die Musterweiterbildungsordnung von 2003 und ihr folgend die aktuelle Weiterbildungsordnung der Ärztekammer Nordrhein aus 2005/2008, zuletzt geändert im März 2014, stellt nur noch auf den Erwerb von Kenntnissen, Erfahrungen und Fertigkeiten ab. Die Strahlentherapie ist in dem nun wieder als „Radiologie“ benannten Gebiet überhaupt nicht mehr erwähnt. Sowohl nach dem zum Zeitpunkt der Approbationserteilung geltenden, als auch nach der aktuellen Weiterbildungsordnung war bzw. ist die Strahlentherapie für den (klagenden) Facharzt für Diagnostische Radiologie damit als fachfremd anzusehen.

\section{Vereinbarkeit mit der Berufsaus- übungsfreiheit (Art. 12 Abs. 1 GG)}

Das Bundesverfassungsgericht (BVerfG) hat wiederholt klargestellt, dass in der (sanktionsbewährten) Beschränkung auf das Fachgebiet grundsätzlich kein unzumutbarer Eingriff in die grundgesetzliche verbürgte Berufsfreiheit des betreffenden Arztes (Art. 12 Abs. 1 GG) gesehen werden kann. Sie ist zumutbar, wenn die Abgrenzung der Bereiche vom fachlich medizinischen Standpunkt aus sachgerecht ist und der Facharzt in der auf sein Fachgebiet beschränkten Tätigkeit eine ausreichende Lebensgrundlage 
finden kann (sog. „Facharztbeschluss“, BVerfGE 33, 125). Die Begrenzung der Facharzttätigkeit auf das eigene Fachgebiet verfolgt das Ziel, die Qualität der fachärztlichen Tätigkeit zu sichern. Demzufolge beruht sie auf vernünftigen Gründen des Gemeinwohls und rechtfertigt demgemäß die Einschränkung der freien Berufsausübung (zuletzt BVerfG NZS 2012, 62).

In seinem Beschluss vom 16.7.2004 (BVerfG NZS 2005, 91) hatte sich das Gericht speziell mit der Frage zu befassen, ob die Verpflichtung zur Beachtung der Fachgebietsgrenzen auch in der vertragsärztlichen Versorgung Geltung entfaltet. Das BVerfG entschied, dass es verfassungsrechtlich nicht zu beanstanden ist, dass (die Sozialgerichtsbarkeit) zur Abgrenzung abrechnungsfähiger ärztlicher Leistungen auf die für das jeweilige Fachgebiet in der Weiterbildungsordnung genannten Inhalte und Ziele der Weiterbildung und die dort genannten Bereiche, in denen eingehende Kenntnisse, Erfahrungen und Fertigkeiten erworben werden müssen, abstellt. Die Inhalte und Ziele der Weiterbildung konkretisieren die allgemeinen Gebietsdefinitionen und geben die speziellen Anforderungen an die Weiterbildung vor. Es kann nach Ansicht des BVerfG zur Sicherung von Qualität und Wirtschaftlichkeit in der gesetzlichen Krankenversicherung sogar eine Beschränkung auf einen engeren Bereich zulässig sein, für den die Weiterbildungsordnung eingehende Kenntnisse, Erfahrungen und Fertigkeiten vorschreibt.

\section{Kein geringfügiger Anteil fachge- bietsfremder Leistungen in der GKV}

In einem nachfolgenden Beschluss (BVerfG NZS 2012, 62), der eine privatärztliche Tätigkeit zugrunde lag, modifizierte das BVerfG seine Auffassung. Ein „systematisches " fachgebietsübergreifendes Tätigwerden ist danach nicht zu beanstanden, insofern dies einen bestimmten Umfang nicht überschreitet. Es ist zu tolerieren, wenn der Anteil der fachfremden Leistungen unter $5 \%$ liegt und sich damit im geringfügigen Bereich bewegt. Die gegenteilige Sichtweise ist nicht mit dem Grundsatz der Verhältnismäßigkeit vereinbar und verstößt daher gegen Art. 12 Abs. 1 GG. Die in der GKV geltende Beschränkung der vertragsärztlichen Abrechnung auf das jeweilige Fachgebiet unter dem Gesichtspunkt der Wirtschaftlichkeit der Versorgung ließ das BVerfG jedoch ausdrücklich unberührt.

Gleichwohl wurden in der Folgezeit Überlegungen dahingehend angestellt, dass auch im vertragsärztlichen Bereich jeder Arzt bis zu $5 \%$ seiner Leistungen aus einem anderen Fachgebiet erbringen und abrechnen darf. Dieser Sichtweise hat das BSG mit dem vorliegenden Urteil eine Absage erteilt. Die Qualifizierung bestimmter Leistungen als fachfremd mit der Folge des Verbotes, sie vertragsärztlich zu erbringen und abzurechnen, ist mit Art. 12 Abs. 1 GG vereinbar. Zwar kann dadurch die berufliche Betätigungsfreiheit berührt sein. Sie betrifft aber nur Leistungen, die nicht in den Kernbereich ihres Fachgebiets fallen bzw. für das Fachgebiet nicht wesentlich und nicht prägend sind. Deren Ausgrenzung ist bei Abwägung zwischen der Eingriffsintensität und den der Tätigkeitsbeschränkung zugrunde liegenden Gemeinwohlbelangen, namentlich die fachkompetente Aufteilung fachärztlicher Zuständigkeiten mit Übersichtlichkeit für die anderen Ärzte und die Patienten sowie des Gesundheitsschutzes, von vernünftigen Gründen des Gemeinwohls gedeckt.

\section{Verhältnis zwischen Weiterbil- dungsordnung und Qualitätssiche- rungsvereinbarungen nach $\S 135$ Abs. 2 SGB V}

Die Ausfassung des BSG, wonach eine Genehmigung der Durchführung und Abrechnung strahlentherapeutischer Leistungen in jedem Fall selbst dann ausscheidet, wenn der Vertragsarzt die Voraussetzungen nach der StrahlendiagV erfüllen würde, weil die Leistungen für den betreffenden Vertragsarzt fachfremd sind, wirft die Frage nach dem prinzipiellen Verhältnis der ärztlichen Weiterbildungsordnung zu den Qualitätssicherungsvereinbarungen nach §135 Abs. 2 SGB $V$ auf. Mithin stellt sich die Frage, wie der Sachverhalt zu beurteilen ist, wenn der Arzt zwar nicht über eine entsprechende Fachgebietsbezeichnung verfügt, gleichzeitig aber die Voraussetzungen eines Ausnahmetatbestandes in §9 Abs. 1 StrahlendiagV erfüllt. Mit Blick auf den Wortlaut der Norm wäre denkbar, dass die Vereinbarung zur Strahlendiagnostik und -therapie einen Erlaubnisvorbehalt zum Verbot der Überschreitung der Fachgebietsgrenzen darstellt. § 9 StrahlendiagV regelt gerade, dass und wie bei fehlender Facharztbezeichnung die erforderliche Qualifikation anderweitig nachgewiesen werden könne. Das BSG ist dieser Sichtweise nicht gefolgt und löst die Normenkollision - ohne nähere Begründung - dahingehend auf, dass in der Vereinbarung zur Strahlendiagnostik und -therapie Qualitätsanforderungen für die Strahlentherapie formuliert werden, deren Vorliegen notwendige, aber noch nicht hinreichende Voraussetzung für eine rechtmäßige Erbringung strahlentherapeutischer Leistungen im Rahmen der vertragsärztlichen Versorgung ist. Zu diesem Ergebnis gelangt auch die obergerichtliche Rechtsprechung. Demgemäß vermag eine Genehmigung nach $\S 2$ StrahlendiagV, die eine besondere Qualifikation bestätigt, die Fachgebietsgrenzen nicht aufzuheben.

Die Fachgebietsumgrenzungen können weder durch besondere persönliche Qualifikationen, durch Sondergenehmigungen der KVen zur Erbringung und Abrechnung weiterer Leistungen, noch durch berufsrechtliche Berechtigungen zur Führung von Zusatzbezeichnungen erweitert werden. Vielmehr ist die Bindung des Arztes an die Grenzen des Fachgebietes, für das er zur Teilnahme an der vertragsärztlichen Versorgung zugelassen ist, auch bei der Erteilung von Genehmigungen nach § 2 Strahlendiag $\vee$ zu beachten, unbeschadet dessen, dass dieses Erfordernis dort nicht ausdrücklich normiert ist (LSG Baden-Württemberg, Urt. v. 8.11.2006, Az.: L 5 KA 1894/05; sich anschließend LSG Nordrhein-Westfalen, Urt. v. 28.5.2015, Az.: L 11 KA 36/11).

\section{Fazit}

Für die Zuordnung bestimmter Leistungen zu einem Fachgebiet kommt es entscheidend darauf an, ob sich diese Leistungen unter die Weiterbildungsinhalte der jeweiligen Weiterbildungsordnung subsummieren lassen, nach der ein Arzt eine Facharztbezeichnung erworben hat. Aufgrund der sich im Laufe der Zeit ändernden Inhalte und Ziele der Weiterbildung in dem jeweiligen Fachgebiet können sich auch die Fachgebietsgrenzen verändern. Das BSG hat in dieser Entscheidung noch einmal betont, dass Beschränkungen des Fachgebietes den Arzt auch in seiner Tätigkeit als Vertragsarzt betreffen. Das Urteil klärt jedoch den Widerspruch zwischen der Weiterbil- 
dungsordnung einerseits und den Qualitätssicherungsvorgaben auf der Grundlage des $§ 135$ Abs. 2 SGB V andererseits nicht vollständig auf. Der betreffende Arzt muss folglich stets dem entsprechenden Fachgebiet angehören, um Leistungen der diagnostischen Radiologie, der Strahlentherapie bzw. der Nuklearmedizin ausführen und abrechnen zu können. Abgesehen von Notfällen ist eine Leistungserbringung außerhalb des Fachgebietes daher nicht möglich. Sofern die Vereinbarung zur Strahlendiagnostik und -therapie zusätzliche Anforderungen hinsichtlich der fachlichen Befähigung und der apparativen Ausstattung vorsieht, muss er diese ebenfalls erfüllen.

Prof. Dr. Peter Wigge,

Rechtsanwalt

Fachanwalt für Medizinrecht
Dipl. jur. Jan Harald Schütz, LL.M., Wiss. Mitarbeiter

Rechtsanwälte Wigge

Scharnhorststraße 40

48151 Münster

Tel.: ++ 49/251/535950

Fax: ++ 49/251/5359599

E-Mail: kanzlei@ra-wigge.de 